\title{
Compensatory Thrombopoietin Production from the Liver and Bone Marrow Stimulates Thrombopoiesis of Living Rat Megakaryocytes in Chronic Renal Failure
}

\author{
Itsuro Kazama ${ }^{a}$ Yasuhiro Endo ${ }^{c}$ Hiroaki Toyama ${ }^{c}$ \\ Yutaka Ejimac Shin Kurosawa ${ }^{c}$ Yoshimichi Murata ${ }^{a}$ \\ Mitsunobu Matsubarab ${ }^{b}$ Yoshio Maruyama ${ }^{a}$ \\ ${ }^{a}$ Department of Physiology I and bivision of Molecular Medicine, Center for Translational \\ and Advanced Animal Research, Tohoku University Graduate School of Medicine, and \\ 'Department of Anesthesiology, Tohoku University Hospital, Sendai, Japan
}

\section{Key Words}

Chronic renal failure $\cdot$ Megakaryocytes Plasma membrane invaginations $\cdot$ Thrombopoiesis $\cdot$

Thrombopoietin • Uremic bleeding

\begin{abstract}
Background/Aims: Decreased thrombopoiesis has been ascribed a role in the pathogenesis of uremic bleeding in chronic renal failure (CRF). However, serum thrombopoietin (TPO) levels are usually elevated in CRF patients, suggesting increased thrombopoiesis. The aim of this study was to determine the thrombopoietic activity in CRF. Methods: Male Sprague-Dawley rats that underwent $5 / 6$ nephrectomy were used as the model of CRF. Age-matched sham-operated rats were used as controls. Single megakaryocytes were isolated from the rat bone marrow, and their size distribution was examined. Megakaryocyte membrane invaginations were monitored by confocal imaging of di-8-ANEPPS staining, and patch clamp whole-cell recordings of membrane capacitance. TPO gene expression was assessed in various tissues. Results: Circulating platelet counts and the number of large megakaryocytes were increased in the bone marrow of CRF rats. Massive di-8-ANEPPS staining and increased membrane capacitance in large megakaryocytes demonstrated increased membrane invaginations. Unaffected Kv1.3-channel currents per cell surface area demonstrated unaltered channel densities. TPO transcription was
\end{abstract}


decreased in the renal cortex but increased in the liver and bone marrow of CRF rats. Conclusion: Increased thrombopoiesis in CRF was thought to be a reactive mechanism to platelet dysfunction. Increased TPO production from the liver and bone marrow compensated for decreased production from damaged kidneys.

Copyright $\odot 2011$ S. Karger AG, Basel

\section{Introduction}

Chronic renal failure (CRF) has been associated with bleeding complications, defined as uremic bleeding [1]. Uremic bleeding is indicated by prolongation of bleeding times, because the major pathogenesis is platelet dysfunction with decreased aggregation and impaired adhesiveness [2-4]. Using the ${ }^{75} \mathrm{Se}$-selenomethionine incorporation technique into platelets, Gafter et al. [5] demonstrated decreased thrombopoietic activity in CRF patients. Because circulating platelet counts were also decreased in some CRF patients $[5,6]$, decreased thrombopoiesis has been associated with the pathogenesis of uremic bleeding. However, Sullivan and McDonald [7] found that platelet dysfunction is one of the stimulators of reactive thrombopoiesis, and later reports have actually demonstrated elevated serum thrombopoietin (TPO) levels in CRF patients [6, 8], suggesting increased thrombopoiesis in CRF, although it has not directly been examined in megakaryocytes, the site of thrombopoiesis.

Recent advances in molecular biology have revealed that thrombopoiesis consists of two stages: initial invagination of the megakaryocyte membrane and later proplatelet release from enlarged megakaryocytes [9-11]. Patch clamp studies in living megakaryocytes have further demonstrated that membrane invaginations are best monitored by whole-cell capacitance measurements and confocal imaging of membrane-impermeant fluorescent indicators [12-14]. Here, using these approaches, we demonstrate for the first time that thrombopoietic activity is increased in CRF, which is thought to be a reactive mechanism to impaired platelet function. We also show that TPO production from the liver and bone marrow is increased in CRF, compensating for the decreased production from damaged kidneys.

\section{Methods}

\section{Animal Preparation}

Rats subjected to 5/6 nephrectomy were used as a model of CRF in the present study. Subtotal nephrectomy was performed in male Sprague-Dawley rats weighing 150-180 g (Japan SLC Inc., Shizuoka, Japan) as described in our previous studies $[15,16]$. Briefly, the upper $1 / 3$ and lower $1 / 3$ of the right kidney was ligated to induce infarction. One week later, the left kidney was removed. During the subsequent 8-week recovery period, rats had free access to standard rat chow and water ad libitum, and were maintained in a humidity- and temperature-controlled room on a 12-hour light-dark cycle. Age-matched sham-operated rats were used as controls. At the end of the recovery period, the rats were deeply anesthetized with isoflurane, and then killed by cervical dislocation. Trunk blood was withdrawn for the measurements of serum creatinine levels and platelet counts. Bone marrow was harvested from the femoral bones for megakaryocyte isolation and RNA extraction. Kidney, liver and spleen were removed for RNA extraction. All experimental protocols described in the present study were approved by the Ethics Review Committee for Animal Experimentation of the Tohoku University. 
Megakaryocyte Preparation and Size Distribution

Single megakaryocytes were isolated from rat bone marrow as described in our previous studies [17-19]. Briefly, bone marrow obtained from femoral bones was flushed with standard external solution containing (in $\mathrm{mM}$ ): $\mathrm{NaCl}, 145 ; \mathrm{KCl}, 4.0 ; \mathrm{CaCl}_{2}, 1.0 ; \mathrm{MgCl}_{2}, 2.0$, and HEPES, 5.0 (pH 7.2 adjusted with $\mathrm{NaOH}$ ), and disseminated by repetitive pipetting. After removing large tissue pieces, cells were washed twice by gentle centrifugation for $1 \mathrm{~min}$ and resuspended in the standard external solution. They were maintained at room temperature $\left(22-24^{\circ} \mathrm{C}\right)$ for use within $8 \mathrm{~h}$.

The megakaryocyte suspension was scattered in a chamber placed on the headstage of an inverted microscope (Nikon, Tokyo, Japan). Megakaryocytes were easily distinguishable from other cells by their large size and multilobed nucleus [20]. Bright-field images were acquired from randomly selected $0.1-\mathrm{mm}^{2}$ fields of view (10 views from 6 sham-operated and 6 CRF rats, respectively). Megakaryocyte sizes were determined by cell surface areas calculated from the diameters measured. Then differently sized megakaryocytes were counted in each field, and their frequencies, expressed as percentage of the total megakaryocyte number, were averaged.

\section{Di-8-ANEPPS Staining}

Single megakaryocytes were incubated for $30 \mathrm{~min}$ at room temperature in external solution containing styryl membrane indicator, di-8-ANEPPS (Biotium, Hayward, Calif., USA; final concentration $10 \mu \mathrm{M}$ ). Fluorescent images were taken using a TE 2000-E Nikon Eclipse confocal microscope (Nikon, Tokyo, Japan).

\section{Electrical Setup and Patch Clamp Recordings}

Single megakaryocytes were subjected to standard patch clamp whole-cell recording techniques, using an EPC-9 patch clamp amplifier system (Heka Electronics, Lambrecht, Germany), as described previously [17-19]. The patch pipette resistance was 1-3 $\mathrm{M} \Omega$ when filled with internal (patch pipette) solution containing (in $\mathrm{mM}$ ): $\mathrm{KCl}, 145 ; \mathrm{MgCl}_{2}, 1.0$; EGTA, 10, and HEPES, 5.0 ( $\mathrm{pH} 7.2$ adjusted with $\mathrm{KOH}$ ). After gigaseal formation, we applied suction briefly to the pipette to rupture the patch membrane. The series resistance of whole-cell recordings was maintained below $10 \mathrm{M} \Omega$ during experiments.

Membrane capacitance of megakaryocytes was determined during whole-cell recordings using an EPC-9 amplifier. We employed a sine plus DC protocol using the Lockin amplifier of the Pulse program. An $800-\mathrm{Hz}$ sinusoidal command voltage was superimposed on the holding potential of $-80 \mathrm{mV}$. Specific membrane capacitance was expressed per unit spherical cell surface area. All experiments were carried out at room temperature.

\section{Real-Time RT-PCR}

Total RNAs from freshly isolated renal cortex, outer and inner medulla, liver, spleen, and bone marrow were extracted using the RNeasy mini kit (Qiagen, Hilden, Germany). Firststand cDNA was synthesized from $2 \mu \mathrm{g}$ of total RNA of each tissue in $20 \mu \mathrm{l}$ of reaction mixture using the SuperScript VILO first-strand synthesis kit (Invitrogen, Carlsbad, Calif., USA). The quantitative RT-PCR was carried out using the Applied Biosystems 7500 RealTime PCR System (Life Technologies Inc., Gaithersburg, Md., USA) using SYBR Premix Ex Taq II (Takara Bio, Kyoto, Japan). The quantity of RNA samples was normalized by the expression level of GAPDH. The sequences of the primers are TPO, forward 5'-CCC AAG CAG AAC CTC TCA AC-3', reverse 5'-TCA GGT ATC CAG GGA TTT GG-3' (200 bp), and GAPDH, forward 5'-GGC ACA GTC AAG GCT GAG AAT G-3', and reverse 5'-ATG GTG GTG AAG ACG CCA GTA-3' (143 bp). 
Table 1. Circulating platelet counts

Kazama et al.: Thrombopoiesis in Uremic Rat Megakaryocytes

Other Measurements and Statistical Analyses

Serum creatinine levels were measured using a chemical autoanalyzer (DRI-CHEM 3500V; Fuji, Tokyo, Japan), platelet counts using a blood cell counter (MICROS abc LC-152; Horiba, Tokyo, Japan). Data were analyzed using PulseFit software (HEKA Electronics), IGOR Pro (WaveMetrics, Lake Oswego, Oreg., USA) and Microsoft Excel (Microsoft Corporation, Redmond, Wash., USA) and reported as means \pm SEM. Statistical significance was assessed by two-way ANOVA followed by Dunnett's or Student's t test. A value of $p<0.05$ was considered significant.

\section{Results}

Thrombocytosis in CRF Rats

As previously demonstrated in our studies $[15,16]$, increased serum creatinine levels in CRF rats reflected renal dysfunction (table 1). Due to the controversial reports on decreases/ increases in circulating platelet counts in CRF patients [5, 6, 21, 22], we aimed to examine circulating platelet counts in our rat model of CRF and found that circulating platelet counts were significantly higher in CRF than sham-operated rats (table 1), indicating reactive thrombocytosis.

\section{Megakaryocyte Size Distribution in Sham-Operated and CRF Rats}

Because megakaryocytes are the precursors of platelets, we first examined their morphological differences between sham-operated and CRF rats. Transparent megakaryocytes were excluded from the size distribution, because they represent damaged cells [23]. Only the sizes of normal, intact megakaryocytes that were kept spherical were assessed (fig. 1a). Depending on their sizes, the cells were divided into three groups: small cells (cells with an area $\left.<400 \mu \mathrm{m}^{2}\right)$; intermediate cells $\left(400-1,000 \mu \mathrm{m}^{2}\right.$; fig. la, left), and large cells $\left(>1,000 \mu \mathrm{m}^{2}\right.$; fig. 1a, right). In sham-operated rats, being consistent with a previous finding [14], most of the megakaryocytes were of intermediate size (fig. 1b), with $<10 \%$ being large megakaryocytes. In CRF rats, the number of intermediate megakaryocytes was much smaller than in sham-operated rats (fig. 1b). However, in contrast, the number of large megakaryocytes was significantly greater $(>2$-fold), suggesting that mature megakaryocytes are increased in CRF rats. The numbers of small megakaryocytes were comparable between sham-operated and CRF rats.

Di-8-ANEPPS Staining and Membrane Capacitance Measurements in Intermediate and Large Megakaryocytes

The demarcation membrane system (DMS) provides the additional membranes required for the process of thrombopoiesis in megakaryocytes [13]. At the beginning of DMS, megakaryocyte membrane invaginations are increased [9-11]. In bright-field images, large mega- 


\begin{tabular}{l|l}
\hline Nephron Extra 2011;1:147-156 \\
\hline $\begin{array}{l}\text { DOI: 10.1159/000333018 } \\
\text { Published online: October 22, } 2011\end{array}$ & $\begin{array}{l}\text { @ 2011 S. Karger AG, Basel } \\
\text { www.karger.com/nne }\end{array}$ \\
\hline
\end{tabular}
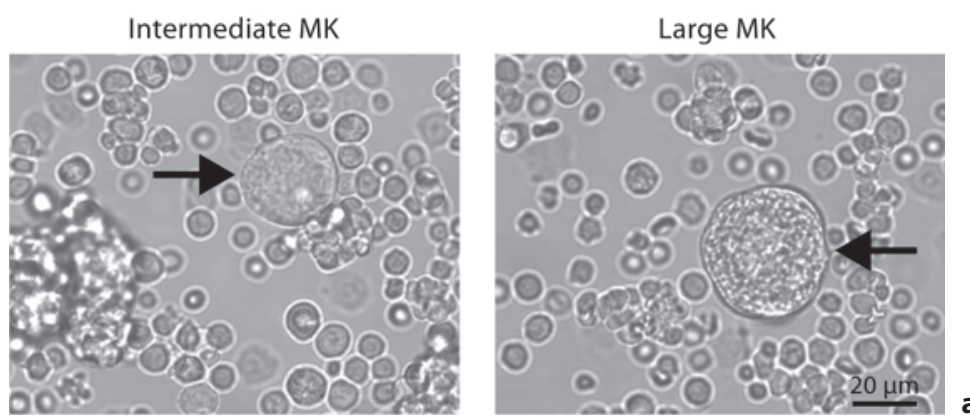

Fig. 1. Megakaryocyte (MK) size distribution in sham-operated (sham) and CRF rats. a Brightfield images of bone marrow cells showing an intermediatesized (left, arrow) and a largesized megakaryocyte (right, arrow). b Frequency histogram of different-size megakaryocytes. The numbers of cells were expressed as percentages of the total megakaryocyte number in selected bright fields. ${ }^{*} \mathrm{p}<0.05$ vs. sham-operated rat bone marrow. Values are means \pm SEM $(\mathrm{n}=$ 60: 10 views from 6 sham-operated and 6 CRF rats, respectively). Differences were analyzed by ANOVA followed by Dunnett's or Student's t test.

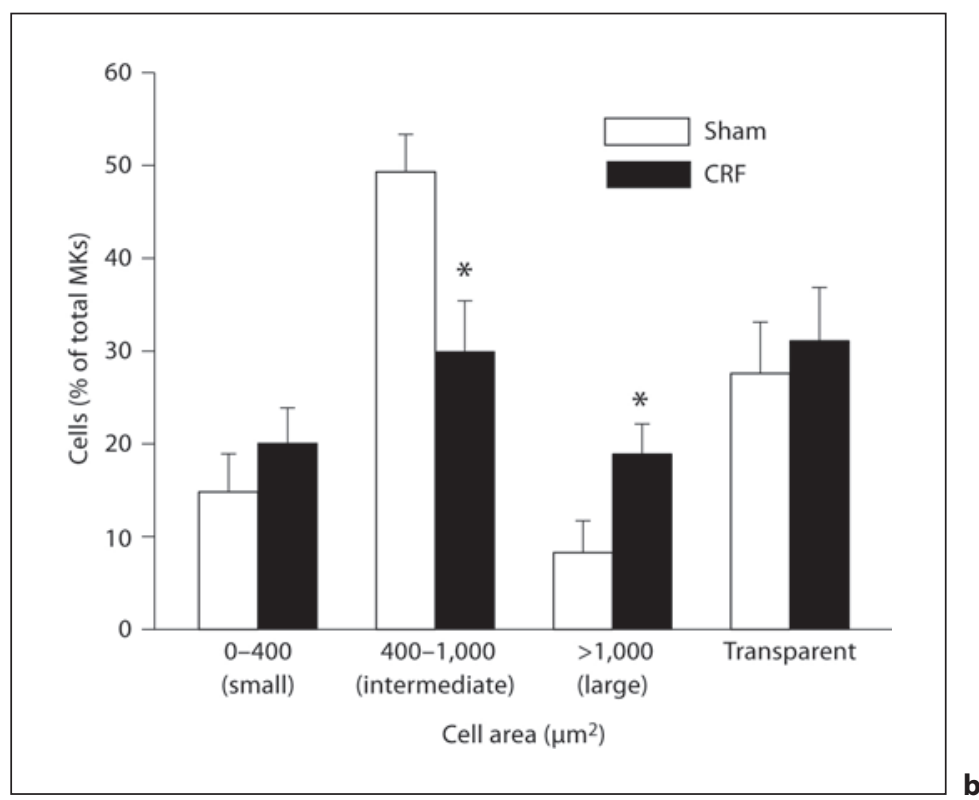

karyocytes appeared to have more wrinkles on their cell surface (fig. 1a, right). To examine whether there are invaginated plasma membranes in the process of thrombopoiesis, we performed di-8-ANEPPS staining [12]. This dye stained peripheral plasma membranes of intermediate megakaryocytes (fig. 2a, left), whereas it penetrated deep into large megakaryocytes and stained the extranuclear cellular volume throughout (fig. 2a, right).

Because specific membrane capacitance expressed per unit spherical cell surface area reflects the amount of invaginated plasma membranes [12, 14], we measured the whole-cell membrane capacitance in megakaryocytes (fig. 2b). Specific membrane capacitance was significantly greater in large than in intermediate megakaryocytes $(12.16 \pm 0.91$ vs. $9.19 \pm$ $\left.0.22 \mu \mathrm{F} / \mathrm{cm}^{2}, \mathrm{p}<0.05\right)$. These results from di-8-ANEPPS staining and membrane capacitance measurements both indicated that invaginated plasma membranes were increased in large megakaryocytes, suggesting increased thrombopoiesis in the bone marrow of the CRF rat.

\section{Kv1.3-Channel Currents in Intermediate and Large Megakaryocytes}

Megakaryocytes are rich in delayed rectifier $\mathrm{K}^{+}$-channels that have recently been identified as Kv1.3 [14]. To examine whether increased membrane invaginations affect Kv1.3-channel currents in megakaryocytes, we conducted whole-cell patch clamp recordings of the currents in intermediate and large megakaryocytes (fig. 3). In both sizes of megakaryocytes, voltage steps from a holding potential of $-80 \mathrm{mV}$ activated large transient outward currents 
Fig. 2. Plasma membrane in-
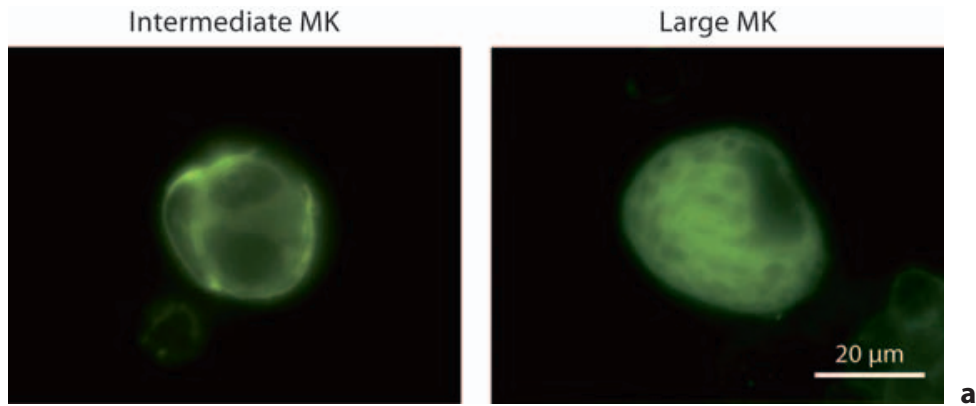
vaginations in intermediate and large megakaryocytes (MK). a Typical confocal fluorescence images of di-ANEPPS-labeled intermediate (left) and large (right) megakaryocytes. b Specific membrane capacitance of megakaryocytes expressed per unit spherical cell surface area. ${ }^{*} \mathrm{p}<0.05$ vs. intermediate megakaryocytes. Values are means \pm SEM $(\mathrm{n}=30)$. Differences were analyzed by ANOVA followed by Dunnett's or Student's t test.

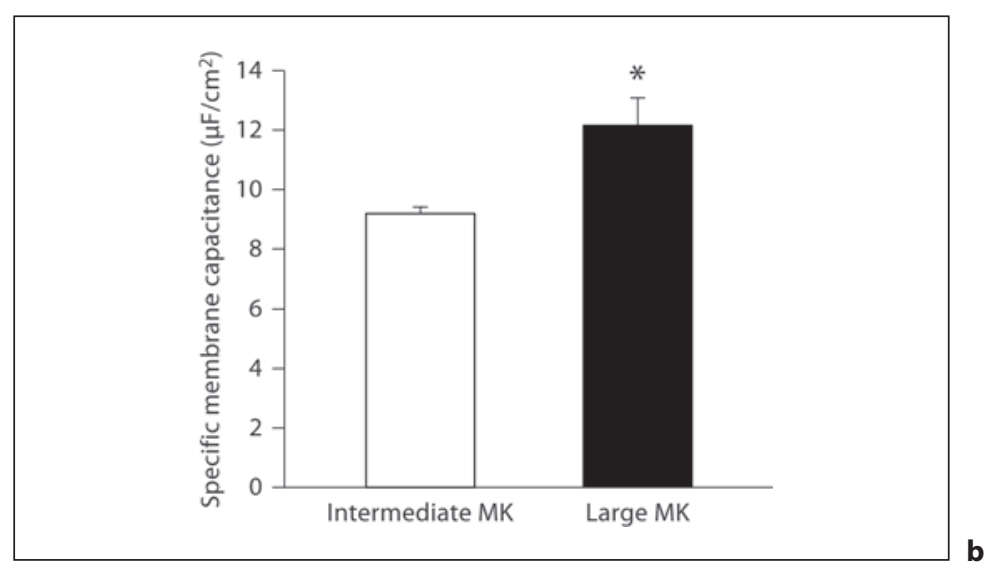

at potentials positive to about $-40 \mathrm{mV}$ (fig. 3a). However, each current amplitude was greater in large megakaryocytes, with a significant difference in a peak current at $+80 \mathrm{mV}(11.78$ \pm 1.20 vs. $7.42 \pm 0.83 \mathrm{nA}, \mathrm{p}<0.05$; fig. $3 \mathrm{~b}$ ). Then, we normalized the peak currents by spherical cell surface areas of megakaryocytes (fig. 3c), only to find the significant difference was lost. From these results, total Kv1.3-channel currents in large megakaryocytes were increased with increasing membrane invaginations. However, the currents per cell surface area were unaffected, indicating unaltered channel densities.

\section{Tissue TPO mRNA Abundance in Sham-Operated and CRF Rats}

TPO is a growth factor that stimulates megakaryocyte maturation and promotes thrombopoiesis [24]. Because the localization of its mRNA expression indicates the site of its synthesis, we examined TPO mRNA abundance in various organs, including the kidney, liver, spleen and bone marrow in sham-operated rats (fig. 4a). As previously demonstrated [25], the highest expression was noted in the liver, followed by the renal cortex, and to a lesser extent the renal medulla, spleen and bone marrow. These results indicate that the liver was the major site of TPO production, and the renal cortex was another one.

In CRF patients, serum TPO levels are elevated $[6,8]$, indicating increased tissue TPO production. To determine the organs that are responsible for the TPO increase, we examined TPO mRNA expression in the renal cortex, liver, spleen and bone marrow from CRF rats, and results were compared with those in sham-operated rats (fig. $4 \mathrm{~b}$ ). In the renal cortex, TPO mRNA expression was significantly lower in CRF rats than in sham-operated rats (fig. 4b, top left), indicating impaired TPO production from damaged kidneys. However, in the liver and bone marrow, TPO mRNA expression was significantly higher in CRF rats than in sham-operated rats (fig. $4 \mathrm{~b}$, top right and bottom right). In the spleen, its abundance was not significantly different between CRF and sham-operated rats (fig. $4 \mathrm{~b}$, bottom left). 

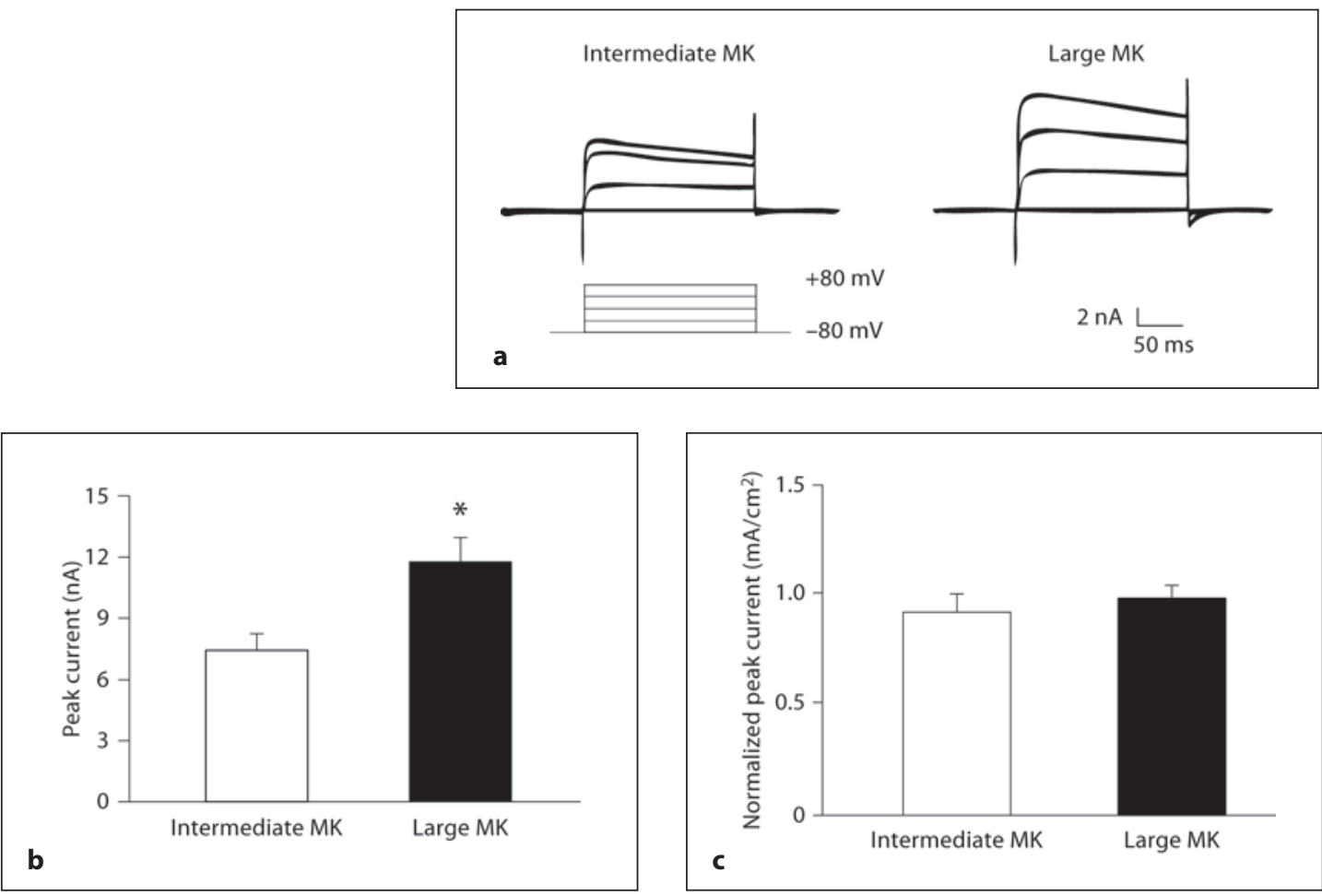

Fig. 3. Kv1.3-channel currents in intermediate and large megakaryocytes (MK). a Typical whole-cell current traces at different voltages recorded in intermediate (left) and large (right) megakaryocytes. The currents were elicited by voltage steps from the holding potential of -80 to $-40,0,40$ and $80 \mathrm{mV}$, as depicted in the voltage protocol. Each pulse was applied for a 200 -ms duration between 10 -second intervals. b Peak current amplitudes for a voltage step to $80 \mathrm{mV}$ obtained from the records in a. c Normalized peak currents expressed per unit spherical cell surface area of megakaryocytes. ${ }^{*} \mathrm{p}<0.05$ vs. intermediate megakaryocytes. Values are means $\pm \operatorname{SEM}(n=30)$. Differences were analyzed by ANOVA followed by Dunnett's or Student's t test.

These results suggested that the liver and bone marrow were responsible for increased TPO production in CRF, and they compensated for decreased TPO production from damaged kidneys.

\section{Discussion}

In the present study, the number of large megakaryocytes was increased in the bone marrow of CRF rats (fig. 1). Membrane capacitance per cell surface area was higher in large than intermediate megakaryocytes (fig. 2b). These findings provided electrophysiological evidence for increased membrane invaginations. Because invagination of the plasma membrane is an initial step of DMS in the process of thrombopoiesis [9-11, 13], our results suggested increased thrombopoietic activities in CRF rats. Previously, using the ${ }^{75} \mathrm{Se}$-selenomethionine incorporation technique into platelets, decreased thrombopoietic activity has been demonstrated in CRF patients [5]. However, this approach was not a direct way to examine megakaryocyte activity. Moreover, results were not consistent with later findings that demonstrated elevated TPO levels in CRF patients $[6,8]$. In the present study, by directly monitoring plasma membrane invaginations of megakaryocytes, we demonstrated increased 
Fig. 4. TPO mRNA abundance in sham-operated and CRF rats. a TPO mRNA abundance in the kidney [cortex $(\mathrm{CX})$ and outer $(\mathrm{OM}) /$ inner medulla (IM)], liver, spleen and bone marrow in sham-operated rats. b Comparison of tissue TPO mRNA abundance between sham-operated (sham) and CRF rats in the renal cortex (top, left), liver (top, right), spleen (bottom, left) and bone marrow (bottom, right). ${ }^{*} \mathrm{p}<0.05$ vs. sham-operated rats. Values are means $\pm \operatorname{SEM}(n=6)$. Differences were analyzed by ANOVA followed by Dunnett's or Student's t test.
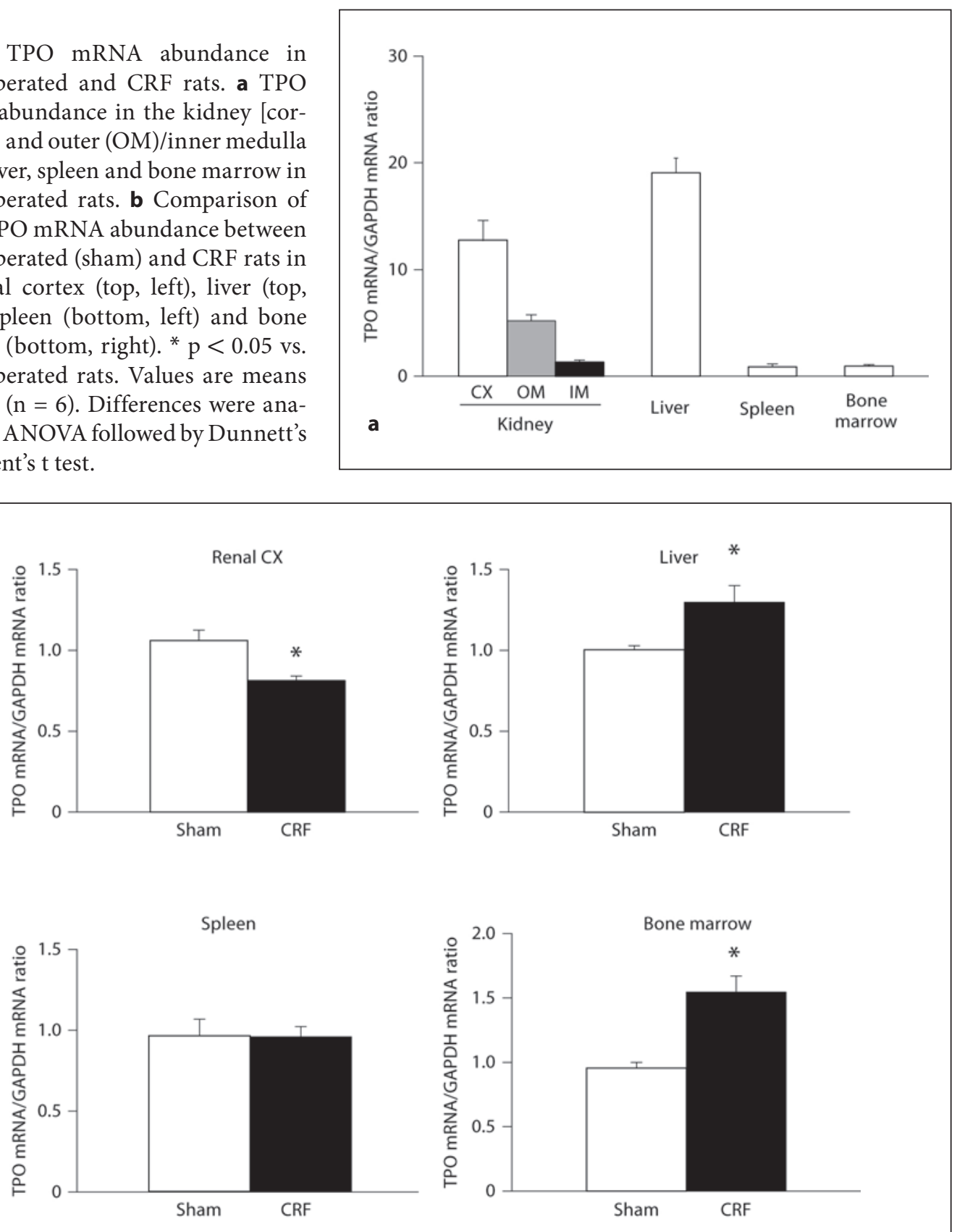

thrombopoiesis in CRF rats. It was considered to be a reactive mechanism to platelet dysfunction caused by uremic toxins in CRF.

Boguslowicz et al. [26] observed dominant distribution of small megakaryocytes and an increased nuclear/cytoplasmic ratio in CRF rat bone marrow. In the present study, although the number of small megakaryocytes was slightly increased in the bone marrow of CRF compared to sham-operated rats (fig. 1b), the increase in large megakaryocytes was more marked. At the beginning of thrombopoiesis, megakaryocytes are enlarged as a result of increased membrane invaginations. Then, after releasing platelets from their cytoplasm, the mega- 
karyocytes finally reduce their size [9-11]. Therefore, both small megakaryocytes in their study and large megakaryocytes in our study represented cells with increased thrombopoietic activity. Because megakaryocytes were alive in our cell preparation, some of them initiated thrombopoiesis in a chamber, presenting large appearance.

In the present study, although membrane invaginations were increased in large megakaryocytes, Kv1.3-channel currents per cell surface area were not affected (fig. 3c). This is consistent with a previous finding that the absence of Kv1.3-channels in megakaryocytes did not affect thrombopoiesis [14]. Because cellular $\mathrm{K}^{+}$efflux can be a driving force for $\mathrm{Ca}^{2+} \mathrm{en}-$ try into the cell, the increased outward $\mathrm{K}^{+}$currents through the channels will elevate cytosolic $\mathrm{Ca}^{2+}$ concentrations of megakaryocytes [17]. Therefore, unaltered Kv1.3-channel currents per cell surface area in large megakaryocytes indicated that the membrane invaginations occurred without the increase in cytosolic $\mathrm{Ca}^{2+}$ concentrations. In our previous study, we have demonstrated that membrane amphipaths, such as salicylate and chlorpromazine, induce plasma membrane curvature changes and, thus, membrane invaginations of megakaryocytes [27]. In CRF rats, some of the uremic toxins may work like these membrane amphipaths and directly increased megakaryocyte membrane invaginations without affecting cytosolic $\mathrm{Ca}^{2+}$ concentrations.

Tissue TPO production is usually controlled by circulating platelet counts [28]. According to Sungaran et al. [25], decreased platelet counts will stimulate bone marrow TPO production and, thus, elevate serum TPO levels. In the present study, however, despite the increase in circulating platelet counts (table 1), both bone marrow and hepatic TPO production was increased in CRF rats (fig. 4b). These results indicated that local TPO production in CRF rats was not likely to be controlled by circulating platelet counts. As a molecular mechanism of reactive thrombocytosis, proinflammatory cytokines such as IL-6 are known to stimulate hepatic TPO production [29]. Since CRF is in a state of chronic inflammation with elevated serum cytokine levels [30], IL-6 may work as a stimulator of both hepatic and bone marrow TPO production in CRF rats.

In conclusion, by monitoring plasma membrane invaginations of CRF rat megakaryocytes, this study demonstrated increased thrombopoietic activity in CRF. It was considered to be a reactive mechanism to platelet dysfunction caused by uremic toxins. Increased TPO production from the liver and bone marrow compensated for the decreased production from the damaged kidneys.

\section{References}

1 Deykin D: Uremic bleeding. Kidney Int 1983;24:698-705.

2 Weigert AL, Schafer AI: Uremic bleeding: pathogenesis and therapy. Am J Med Sci 1998;316:94-104.

3 Escolar G, Cases A, Bastida E, Garrido M, Lopez J, Revert L, et al: Uremic platelets have a functional defect affecting the interaction of von Willebrand factor with glycoprotein IIb-IIIa. Blood 1990;76: $1336-1340$.

4 Gawaz MP, Dobos G, Spath M, Schollmeyer P, Gurland HJ, Mujais SK: Impaired function of platelet membrane glycoprotein IIb-IIIa in end-stage renal disease. J Am Soc Nephrol 1994;5:36-46.

5 Gafter U, Bessler H, Malachi T, Zevin D, Djaldetti M, Levi J: Platelet count and thrombopoietic activity in patients with chronic renal failure. Nephron 1987;45:207-210.

6 Linthorst GE, Folman CC, van Olden RW, von dem Borne AE: Plasma thrombopoietin levels in patients with chronic renal failure. Hematol J 2002;3:38-42.

7 Sullivan PS, McDonald TP: Acetylsalicylic acid stimulates murine megakaryocyte precursor cells. Proc Soc Exp Biol Med 1990;194:216-220.

8 Otsubo Y, Nitta K, Otsubo S, Kimata N, Miwa N, Watanabe Y, et al: Circulating levels of thrombopoietin and erythropoietin in hemodialysis patients. Acta Haematol 2003;109:209-211. 
9 Patel SR, Hartwig JH, Italiano JE Jr: The biogenesis of platelets from megakaryocyte proplatelets. J Clin Invest 2005;115:3348-3354.

10 Kosaki G: Platelet production by megakaryocytes: protoplatelet theory justifies cytoplasmic fragmentation model. Int J Hematol 2008;88:255-267.

11 Thon JN, Montalvo A, Patel-Hett S, Devine MT, Richardson JL, Ehrlicher A, et al: Cytoskeletal mechanics of proplatelet maturation and platelet release. J Cell Biol 2010;191:861-874.

12 Mahaut-Smith MP, Thomas D, Higham AB, Usher-Smith JA, Hussain JF, Martinez-Pinna J, et al: Properties of the demarcation membrane system in living rat megakaryocytes. Biophys J 2003;84: 2646-2654.

13 Schulze H, Korpal M, Hurov J, Kim SW, Zhang J, Cantley LC, et al: Characterization of the megakaryocyte demarcation membrane system and its role in thrombopoiesis. Blood 2006;107:38683875.

14 McCloskey C, Jones S, Amisten S, Snowden RT, Kaczmarek LK, Erlinge D, et al: Kv1.3 is the exclusive voltage-gated $\mathrm{K}^{+}$channel of platelets and megakaryocytes: roles in membrane potential, $\mathrm{Ca}^{2+}$ signalling and platelet count. J Physiol 2010;588:1399-1406.

15 Michimata M, Kazama I, Mizukami K, Araki T, Nakamura Y, Suzuki M, et al: Urinary concentration defect and limited expression of sodium cotransporter, $\mathrm{rBSC} 1$, in a rat model of chronic renal failure. Nephron Physiol 2003;93:34-41.

16 Suzuki K, Hatano R, Michimata M, Kazama I, Suzuki M, Miyama N, et al: Residual urinary concentrating ability and AQP2 expression in a rat model for chronic renal failure. Nephron Physiol 2005; 99:16-22.

17 Ikeda M, Kurokawa K, Maruyama Y: Cyclic nucleotide-dependent regulation of agonist-induced calcium increases in mouse megakaryocytes. J Physiol 1992;447:711-728.

18 Shimada H, Tomita Y, Inooka G, Maruyama Y: Sodium-coupled neutral amino acid cotransporter inhibited by the volatile anesthetic, halothane, in megakaryocytes. Jpn J Physiol 1995;45:165-176.

19 Ikeda M, Kurokawa K, Maruyama Y: $\mathrm{Ca}^{2+}$ spike initiation from sensitized inositol 1,4,5-trisphosphate-sensitive $\mathrm{Ca}^{2+}$ stores in megakaryocytes. Pflugers Arch 1994;427:355-364.

20 Mahaut-Smith MP: Patch-clamp recordings of electrophysiological events in the platelet and megakaryocyte. Methods Mol Biol 2004;273:277-300.

21 Sokunbi D, Wadhwa NK, Solomon M, Suh H: Thrombocytosis in diabetic and nondiabetic end-stage renal disease patients on peritoneal dialysis. Adv Perit Dial 1993;9:156-160.

22 Mesquita Mdo C, Sol EB, Malarme M, Noubouossie D, Demulder AC: Management of refractory essential thrombocythemia with anagrelide in a patient undergoing hemodialysis. Clin Ther 2009;31: 2559-2564.

23 Carter RN, Tolhurst G, Walmsley G, Vizuete-Forster M, Miller N, Mahaut-Smith MP: Molecular and electrophysiological characterization of transient receptor potential ion channels in the primary murine megakaryocyte. J Physiol 2006;576:151-162.

24 Kuter DJ, Begley CG: Recombinant human thrombopoietin: basic biology and evaluation of clinical studies. Blood 2002;100:3457-3469.

25 Sungaran R, Markovic B, Chong BH: Localization and regulation of thrombopoietin mRNA expression in human kidney, liver, bone marrow, and spleen using in situ hybridization. Blood 1997;89: 101-107.

26 Boguslowicz W, Lemancewicz D, Pawlak D, Dzieciol J: Histomorphometry of marrow megakaryocytes in experimental uraemia in rats. Rocz Akad Med Bialymst 2004;49(suppl 1):159-160.

27 Kazama I, Murata Y, Maruyama Y: Effects of membrane amphipath on delayed rectifier $\mathrm{K}^{+}$-channel currents in megakaryocytes. J Physiol Sci 2011;61(suppl 1):S215.

28 Kuter DJ, Rosenberg RD: The reciprocal relationship of thrombopoietin (c-Mpl ligand) to changes in the platelet mass during busulfan-induced thrombocytopenia in the rabbit. Blood 1995;85:27202730.

29 Kaser A, Brandacher G, Steurer W, Kaser S, Offner FA, Zoller H, et al: Interleukin-6 stimulates thrombopoiesis through thrombopoietin: role in inflammatory thrombocytosis. Blood 2001;98: $2720-2725$.

30 Barreto DV, Barreto FC, Liabeuf S, Temmar M, Lemke HD, Tribouilloy C, et al: Plasma interleukin-6 is independently associated with mortality in both hemodialysis and pre-dialysis patients with chronic kidney disease. Kidney Int 2010;77:550-556. 